Communaut'ERE - Les défis de l'éducation relative à l'environnement centrée sur la communauté et sur les lieux

Conférence EECOM - Montréal, Québec- 11 au 15 août 2002

Tom Berryman

\title{
OpenEdition
}

Journals

Édition électronique

URL : http://journals.openedition.org/ere/5562

DOI : 10.4000/ere.5562

ISSN : 2561-2271

Éditeur

Centr'ERE

Référence électronique

Tom Berryman, « Communaut'ERE - Les défis de l'éducation relative à l'environnement centrée sur la communauté et sur les lieux », Éducation relative à l'environnement [En ligne], Volume 4 | 2003, mis en ligne le 14 septembre 2003, consulté le 24 septembre 2020. URL : http://journals.openedition.org/ere/ 5562 ; DOI : https://doi.org/10.4000/ere.5562 


\section{Communaut'ERE - Les défis de l'éducation relative à l'environnement centrée sur la communauté et sur les lieux}

Conférence EECOM - Montréal, Québec- 11 au 15 août 2002

Tom Berryman

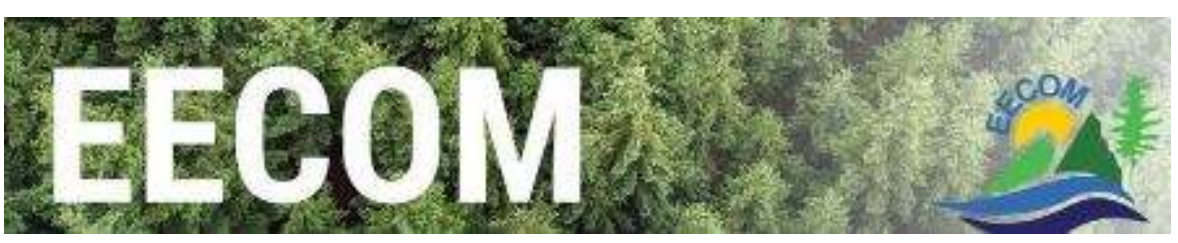

1 Alors qu'un important smog faisait partiellement écran au soleil et que les Montréalais suffoquaient sous des chaleurs records dans un air humide et immobile, quelque cent cinquante personnes ont échangé bien au frais sur les défis de l'éducation relative à l'environnement centrée sur la communauté. C'est dans le confort des salles de l'Université du Québec à Montréal (UQAM) que les participants à la huitième conférence annuelle du Réseau canadien d'éducation et de communication relatives à l'environnement (EECOM) ont pu explorer pendant quatre jours les diverses dimensions d'une éducation relative à l'environnement qui cherche à prendre appui sur la communauté ainsi que sur les lieux. Le seul moment où il plu fut lors de la visite de divers sites éducatifs : parc, ferme, musée et centre d'interprétation.

2 Provenant de milieux, de régions et d'horizons culturels très variés, les personnes présentes à la conférence EECOM pouvaient communiquer leur propos en anglais ou en français afin de faire le point sur les réalisations, les défis, les enjeux et les possibles pièges de ce que les anglophones nomment le «community and place-based environmental education ». La principale organisation responsable de la rencontre, l'Association québécoise pour la promotion de l'éducation relative à l'environnement (AQPERE), s'est presque exclusivement centrée sur les liens dans des réseaux 
francophones au cours des 10 dernières années. Cette conférence offrait enfin une occasion d'élargir les horizons en tentant de s'approcher, à l'aide notamment de la traduction simultanée, de nos voisins immédiats sur le continent nord-américain. En effet, jusqu'ici, nombre de francophones du Québec avaient davantage de liens avec des Français, des Belges et des francophones de l'Afrique qu'ils n'en avaient avec leurs voisins immédiats du Canada et des États-Unis.

3 L'un des défis des organisateurs, outre les traditionnelles difficultés lors des rencontres entre ceux qui parlent la langue de Shakespeare et ceux qui parlent la langue de Molière, était de favoriser un décloisonnement entre les aspects théoriques et pratiques, entre les recherches en éducation et les pratiques éducatives variées. Rappelons en effet qu'en plus de l'AQPERE et d'EECOM, la conférence était aussi organisée avec le support de la Chaire de recherche du Canada en éducation relative à l'environnement et celui de la Biosphère d'Environnement Canada. Le programme visait autant que possible à ne pas cloisonner les diverses dimensions de l'éducation relative à l'environnement. Néanmoins, au bilan, certains éducateurs ont considéré que la conférence était trop théorique et pas assez «pratico-pratique». D'autre part, certains chercheurs ont quant à eux déploré que trop de communications illustraient parfois un manque de recul sur la pratique et constituaient en quelque sorte de la promotion. Cette polarisation, assez classique, illustre néanmoins qu'il devait y avoir lors de la conférence du contenu pour interpeller presque toutes les personnes présentes, mais que la rencontre effective entre les perspectives théoriques et pratiques demeure hélas encore difficile.

4 De plus, même si les participants ont effectivement pu échanger librement autour des questions portant sur les réalisations, les défis, les enjeux et les possibles pièges des pratiques d'éducation relative à l'environnement prenant appui sur la communauté et sur les lieux, ce qui est déjà beaucoup, on peut tout de même déplorer, au terme de la rencontre, un manque de clarification des concepts clés derrière les mots «lieu » et " communauté ». Cette conférence aurait probablement dès lors bénéficié d'une session plénière consacrée à une tentative de clarification des quelques notions clés au coeur de la thématique. Déplorons aussi que l'organisation n'ait pu se doter des moyens nécessaires afin de produire des actes. C'est toutefois en considérant attentivement l'ampleur du travail nécessaire à la production d'actes de qualité, au regard des moyens limités qui lui étaient alloués, que ce choix fut fait.

5 Quoiqu'il en soit, la perspective critique était tout de même au rendez-vous et la conférence EECOM fut ainsi une belle occasion de se pencher de manière plus éclairée sur cette ré-émergence des questions communautaires et sur l'importance de l'esprit des lieux (genius loci) en éducation relative à l'environnement. Ici, pas d'imitation des joutes diplomatiques et des négociations autour d'énoncés constituant une nouvelle grande déclaration solennelle. Ici, pas de jeux de négociations en vue d'établir un vaste programme commun et global d'action. EECOM était avant tout un moment pour se rencontrer entre éducateurs, pour échanger et pour se former avant de retourner chacun chez-soi, dans nos milieux de vie et de pratique respectifs afin de chercher à mieux y oeuvrer. Les défis de l'éducation relative à l'environnement centrée sur la communauté et sur les lieux demeurent immenses. En effet, si les grandes approches thématiques souvent centrées sur les enjeux socio-environnementaux globaux de l'heure tiennent encore généralement le haut du pavé lorsqu'il est question d'éducation relative à l'environnement, une recentration sur ce qui se passe dans notre milieu de 
vie révèle souvent de grands défis éducatifs afin de se réapproprier et de mieux soigner, individuellement et collectivement, ces différents lieux où se déploient notre être.

\section{AUTEUR}

\section{TOM BERRYMAN}

Université du Québec à Montréal 